\title{
MODELO DE PROGRAMAÇÃO MATEMÁTICA PARA CONTROLE DO CRESCIMENTO DA VEGETAÇÃO SOB REDES DE DISTRIBUIÇÃO DE ENERGIA ELÉTRICA
}

\author{
Liliani A. V. Apolinário* \\ lilianiapolinario@yahoo.com.br
}

\author{
José R. Sanches Mantovani* \\ mantedee.feis.unesp.br
}

\author{
*Departamento de Engenharia Elétrica - Faculdade de Engenharia de Ilha Solteira \\ Universidade Estadual Paulista - UNESP \\ Laboratório de Planejamento de Sistemas de Energia Elétrica, \\ Caixa Postal 31, CEP 15385-000, Ilha Solteira/SP
}

\begin{abstract}
Mathematical Programming Model for Control of Growth of Vegetation in Electrical Power Distribution Networks

In this paper the problem of scheduling execution of tree pruning under the distribution network is formulated as a binary programming model. This model seeks reducing the fault rates due to the vegetation and the optimized allocation of the annual financial and human resources available by the utilities to maintain the trees under the feeders without violating the financial resources constraints and the reliability index. The reliability index due to the vegetation causes are obtained by regression techniques of historical data from the faults occurred in each feeder. To solve the optimization model it is proposed a dedicated Chu-Beasley genetic algorithm. The proposed methodology is tested for an aerial distribution system of a medium size city.
\end{abstract}

KEYWORDS: Vegetation Maintenance, Distribution Systems, Chu-Beasley Genetic Algorithm.

\section{RESUMO}

Neste trabalho o problema da programação de execução de

Artigo submetido em 12/11/2009 (Id.: 01076)

Revisado em 14/02/2010, 01/08/2010, 18/12/2010, 21/01/2011

Aceito sob recomendação do Editor Associado Prof. Ivan Nunes Da Silva podas de árvores sob a rede de distribuição é formulado como um modelo matemático de programação binária. Neste modelo busca-se a redução das taxas de faltas devido à vegetação e a alocação otimizada de recursos humanos e financeiros, anuais, disponíveis pelas empresas distribuidoras para manutenção da vegetação sob a rede, de modo a não violar as restrições financeiras, os recursos humanos disponíveis pelas empresas e os índices de confiabilidade do sistema. Os índices de confiabilidade devido a interferência da vegetação são obtidos através da técnica de regressão dos dados da série histórica das faltas ocorridas devido à vegetação em cada alimentador. Para solução do modelo de otimização proposto utiliza-se um algoritmo genético de Chu-Beasley. A metodologia proposta é validada e testada em um sistema de distribuição aéreo de uma cidade de médio porte.

PALAVRAS-CHAVE: Manutenção da Vegetação, Sistema de Distribuição, Algoritmo Genético de Chu-Beasley.

\section{INTRODUÇÃO}

O sistema de distribuição é predominantemente do tipo aéreo na maioria dos países, principalmente nas regiões localizadas no entorno dos centros comerciais, devido o seu baixo custo, embora com índices de confiabilidade menores. As redes aéreas estão sujeitas à invasão da vegetação que provoca a interrupção dos serviços de fornecimento de energia causando transtornos para os consumidores e 
deteriorando os indicadores de confiabilidade das empresas distribuidoras. A vegetação causa direta ou indiretamente curto-circuito entre os condutores, ou ela pode atuar mecanicamente nos equipamentos da linha. Os galhos de árvores podem cair sobre os condutores, empurrar os condutores aproximando-os e facilitando o contato entre as fases ou estabelecendo a formação do arco elétrico em condições ambientais propícias, e podem servir também como meio de acesso à rede de distribuição para os animais que são os grandes causadores de faltas em sistemas elétricos (Wischkaemper et al., 2008).

Os programas de podas de árvores sob a rede de distribuição são necessários para garantir a segurança e confiabilidade no fornecimento de eletricidade e preservar a qualidade da iluminação pública e de outros serviços urbanos essenciais (CESP, 1995; ?; ?; ?). As podas devem ser realizadas de forma sistemática obedecendo ao ciclo de crescimento das espécies, em que as árvores devem ser cortadas de maneira a manter uma distância mínima entre o condutor elétrico e a extremidade da vegetação, que constitui o chamado "limite de segurança" (Oliveira Junior, 1996; ?; ?).

Na maioria das empresas distribuidoras, o programa atual de manutenção da vegetação em espaços urbanos sob as redes baseia-se numa inspeção externa visual para diagnosticar as características dendrométricas (altura, diâmetro da copa, diâmetro do tronco) das árvores. Se a altura da vegetação for maior que a máxima permitida para não interferir no fornecimento de energia, então é executada a manutenção da vegetação, caso contrário nenhum procedimento é realizado. Este tipo de procedimento pode levar a resultados satisfatórios, mas de qualidade inferior quando comparados com os resultados obtidos de forma otimizada visando a melhoria dos índices de confiabilidade da rede (Billinton and Billinton, 1989; IEEE Std. 1366, 2003) e a alocação de recursos humanos e financeiros disponíveis pelas empresas para este tipo de atividade. A redução dos custos da manutenção da vegetação sob a rede elétrica pode ser obtida tanto pelo plantio de árvores adequadas para dividir o espaço com a rede, como com a adoção de procedimentos otimizados corretos obtidos através de um modelo matemático, que prevê que as atividades de manutenção sejam realizadas em períodos do ano adequados para conservação das árvores de grande porte remanescentes sob a fiação elétrica.

$\mathrm{Na}$ literatura, encontram-se poucos trabalhos relacionados com o tema desenvolvimento de ferramentas matemáticas para auxiliar no planejamento de podas e manutenção da vegetação existente sob as redes elétricas. No modelo proposto em (Kuntz et al., 2002) são consideradas, separadamente, três funções objetivos: (1) Minimizar o custo total da confiabilidade, (2) Minimizar os custos de manutenção para obter um valor de confiabilidade preestabelecido; (3) Maximizar a confiabilidade do sistema para um valor preestabelecido de custo de manutenção. As restrições são a disponibilidade de recursos humanos da empresa, os custos de manutenção e a violação de um valor de confiabilidade preestabelecido. Em (Appelt and Beard, 2006) é apresentado um programa de gerenciamento da vegetação sob as redes de distribuição. A maior dificuldade para modelar a interferência das diferentes espécies de vegetação nos índices de confiabilidade do sistema elétrico está em estabelecer uma relação entre as taxas de faltas e o crescimento da vegetação sob a rede. Em (Radmer et al., 2002) é proposto um modelo para estimar as taxas de faltas permanentes devido à vegetação sob a rede, que é uma extensão do modelo de taxas de faltas obtido através de uma rede neural. No modelo de rede neural busca-se uma relação entre o crescimento da vegetação e as interrupções devido à interferência da vegetação sob a rede para determinar os índices de taxas de faltas permanentes.

Neste trabalho propõe-se um novo modelo de programação matemática para o problema de planejamento otimizado da manutenção de podas de árvores sob as redes de distribuição. Este modelo difere do modelo apresentado em (Kuntz et al., 2002) na sua concepção filosófica que considera uma única função objetivo e um conjunto de restrições que representa de forma realista o problema de manutenção de podas enfrentado pelas empresas distribuidoras. A função objetivo deste modelo contempla a redução dos custos de energia não distribuída devido à interferência da vegetação na rede e a redução dos custos de mão de obra das equipes de manutenção. As restrições consideradas no modelo são a meta preestabelecida dos índices de confiabilidade e os recursos humanos e financeiros disponíveis anualmente pela empresa para as atividades de manutenção (Apolinário, 2009; Apolinário e Mantovani, 2008). Para resolução deste modelo é proposto o Algoritmo Genético de Chu-Beasley (AGCB) (Chu and Beasley, 1997). Adicionalmente a esta seção introdutória, este artigo está organizado da seguinte forma: $\mathrm{Na}$ Seção 2 apresenta-se a concepção filosófica adotada no desenvolvimento do modelo matemático proposto para o planejamento do manejo da vegetação sob a rede de distribuição. Na Seção 3 apresentam-se os detalhes do algoritmo genético de Chu-Beasley dedicado à solução do modelo matemático. Na Seção 4 apresentam-se os testes e os resultados obtidos a partir da implementação computacional da metodologia proposta. Para obtenção desses resultados, que também são discutidos nesta seção, utiliza-se um sistema de distribuição real de uma cidade de médio porte do interior do estado de São Paulo. Na Seção 5 estão as análises e as conclusões relativas tanto quanto à importância como à forma de abordagem proposta para o problema de manutenção da vegetação sob redes de distribuição. 


\section{O PROBLEMA DE MANUTENÇÃO DA VEGETAÇÃO}

O programa de manutenção de podas de árvores sob a rede de distribuição abordado nesta seção compreende à descrição da filosofia adotada para obtenção do modelo matemático, e do parâmetro básico de natureza probabilística envolvido nesse modelo que relaciona as taxas de faltas nas redes de distribuição devido à interferência da vegetação. Apresenta-se também nesta seção as restrições de natureza física e econômica envolvidas na elaboração do modelo.

O modelo matemático do problema de manutenção da vegetação é desenvolvido através de uma função objetivo que considera a redução dos custos de desligamentos da rede devido à vegetação e a alocação otimizada dos recursos humanos e financeiros destinados pelas empresas para efetuar a manutenção da vegetação. A solução deste modelo deve fornecer quando e onde deve ser efetuada a poda da vegetação sob a rede considerando-se um período de planejamento preestabelecido. Genericamente este modelo pode ser descrito como:

\section{Minimizar $\left\{\begin{array}{c}\text { Custo de Manutenção da Vegetação } \\ +\end{array}\right.$}

\subsection{Taxas de Faltas}

Em (Kuntz et al., 2002) são propostas duas metodologias para obter a equação das taxas de faltas em sistemas de distribuição devido à interferência da vegetação. $\mathrm{Na}$ primeira, utilizam-se informações do crescimento da vegetação relacionadas com os tipos de espécies e às condições ambientais e meteorológicas para determinar as taxas de faltas. Na outra metodologia as taxas de faltas são obtidas diretamente dos dados históricos dos índices de confiabilidade dos alimentadores devido à interferência da vegetação. Nesta metodologia, para estimar as taxas de faltas não há a necessidade de um segundo modelo que relaciona o crescimento da vegetação às taxas de faltas, e por consequência não necessitando também que o mecanismo de faltas devido às taxas de crescimento da vegetação esteja explícito no modelo, como ilustrado na Figura 1.

O modelo que usa a taxa de crescimento da vegetação é mais preciso, mas vários problemas aparecem ao aplicá-lo para obter uma estimativa das taxas de faltas. Estes problemas incluem o intervalo de tempo de análise, as várias espécies de vegetação que estão sob a rede e o estabelecimento do modelo matemático adequado para representar o crescimento vertical e radial das árvores. No modelo direto para estimar as taxas de faltas não se necessita de uma equação adicional

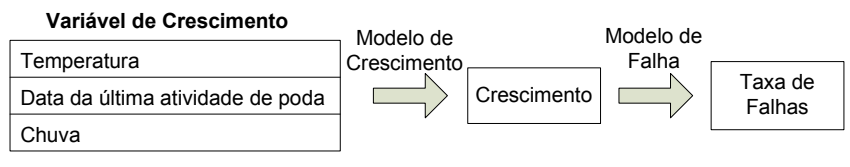

(a) Utiliza modelo de crescimento das espécies

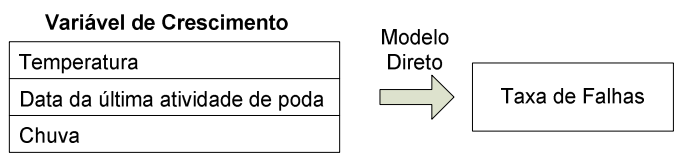

(b) Modelo direto.

Figura 1: Modelos para obtenção das taxas de faltas devido à vegetação.

para fazer a ligação entre o crescimento da vegetação e as taxas de faltas.

Desta forma, devido às dificuldades para obter os dados necessários para o modelo que usa as informações sobre o crescimento da vegetação e as diferentes espécies de plantas sob a rede, propõe-se neste trabalho o modelo direto para estimar as taxas de faltas devido à vegetação.

\subsubsection{Modelos diretos para estimativa das taxas de faltas}

No modelo direto proposto utilizam-se os dados relacionados com as taxas de faltas devido à vegetação que podem ser obtidos na forma de pontos, cujo comportamento demonstra o relacionamento de uma variável independente (ou explicativa) com uma, ou mais, variável dependente (ou resposta). O gráfico destes pontos é chamado de diagrama de dispersão e relaciona as taxas de faltas devido à vegetação em função de um período para análise.

Em (Guikema et al., 2006) são desenvolvidos modelos estatísticos para estimar o impacto da poda de árvores na retirada de serviços de sistemas elétricos operando sob condições ambientais normais, e cosequentemente nas taxas de faltas na rede devido a vegetação. Os modelos usados são um modelo linear binomial negativo generalizado e um modelo linear generalizado misto de Poisson. Estes modelos são recomendados para análise de dados de contagem, ou seja, quando a variável independente deve ser um valor inteiro não-negativo (variável aleatória), como, por exemplo, a retirada de serviço de um circuito ou de um alimentador num intervalo de tempo de um ano.

\subsubsection{Modelo proposto}

Dado um gráfico de dispersão, é pouco provável que haja uma curva que passe exatamente em cada ponto e que descreva fielmente o sistema sob análise. A razão disto é que 
os dados observados possuem erros inerentes ao processo. Isso se deve ao fato de que os dados utilizados em um gráfico de dispersão são normalmente dados obtidos de amostras e, portanto, para que exista uma curva comum entre os pontos (curva de regressão) é necessário que a correlação existente entre os dados seja forte.

Considerando as propostas de modelo encontradas na literatura para estimativas das taxas de faltas no sistema de distribuição devido à vegetação e os dados reais fornecidos por uma empresa distribuidora, neste trabalho defini-se uma função analítica que descreva o comportamento das taxas de faltas em função de um período subsequente, obtendo-se uma curva que melhor se ajuste aos pontos que relacionam as taxas de faltas no período considerado, levando-se em consideração a existência de erros, em geral, que não são previsíveis.

Uma das vantagens de se obter uma curva que se ajusta adequadamente aos dados é a possibilidade de prever os valores da função (variável dependente) para valores da variável explicativa que estão fora do intervalo de tempo que estes dados foram obtidos. Ou seja, é possível fazer uma extrapolação (predição) para um tempo futuro próximo com uma aproximação razoável. Desta forma propõem-se modelos não-lineares para a estimativa das taxas de faltas, que são dados pela seguinte equação geral:

$$
Y_{i}=g\left(X_{i}, \beta\right)+\varepsilon_{i}
$$

sendo:

$g($.$) : Função não linear, cujo modelo de distribuição de$ probabilidades depende da base de dados de faltas no alimentador devido a vegetação;

$\varepsilon_{i}:$ Erro;

$\beta$ : Vetor de parâmetros do modelo relacionado com a distribuição temporal das variáveis independentes;

$X_{i}$ : Variável independente ou explicativa (aleatória).

Este modelo pode ser utilizado para obter as equações das taxas de faltas devido à vegetação para todos os alimentadores da rede de distribuição. Os dados disponíveis para cada alimentador apresentam uma equação que melhor se ajusta aos mesmos e permita fazer uma previsão das taxas de faltas para o período em estudo.

\subsection{Modelo Matemático}

O modelo de função objetivo proposto visa minimizar os custos de manutenção da vegetação e melhorar a confiabilidade da rede de distribuição de modo a reduzir o máximo possível as ocorrências de faltas no sistema devido à vegetação urbana. Desta forma, a função objetivo é formulada considerando-se os custos da energia não suprida devido à interferência da vegetação e os custos de manutenção em cada período de planejamento. As restrições devem contemplar as limitações de recursos disponíveis e atender as metas das empresas com relação aos índices de desligamentos devido a interferência da vegetação. O modelo matemático proposto para o problema é dado por:

$$
\begin{aligned}
& \text { minimizar } \\
& C=C_{0}+\sum_{k=1}^{N A} \sum_{t=1}^{T}\left[\begin{array}{l}
\left(D R_{k} \% \cdot C_{\mathrm{CR}}+D I_{k} \% \cdot C_{\mathrm{CI}}\right. \\
\left.+D C_{k} \% \cdot C_{\mathrm{CC}}\right) \\
\left(\begin{array}{l}
T R_{k} \\
\left.\sum_{t r=1} D(k, t r) \cdot P(k, t r)\right) \\
+\left(C_{\operatorname{man}} \cdot l(k, t)\right) r(k, t)
\end{array}\right) \lambda(k, t)
\end{array}\right]
\end{aligned}
$$

sujeito a:

$$
\begin{aligned}
& \sum_{k=1}^{N A} \sum_{t=1}^{T} C_{\operatorname{man}} \cdot l(k, t)-C_{\text {esp }} \leq 0 \\
& \sum_{k=1}^{N A} l(k)-L_{\text {esp }}^{t} \leq 0 \quad \text { para } \mathrm{t}=1, \cdots, \mathrm{T} \\
& F E C-M F \leq 0 \\
& C_{0}=\sum_{k=1}^{N A}\left[\begin{array}{l}
\left(D R_{k} \% \cdot C_{\mathrm{CR}}+D I_{k} \% \cdot C_{\mathrm{CI}}+D C_{k} \% \cdot C_{\mathrm{CC}}\right) \\
\left.\left(\begin{array}{l}
\sum_{t r} R_{k} \\
t r=1 \\
+\left(C_{\operatorname{man}} l(k, 0)\right)
\end{array}\right) \cdot P(k, t r)\right) \lambda(0, k)
\end{array}\right.
\end{aligned}
$$

sendo:

$C_{0}$ : Custo da energia não suprida pela rede devido à interferência da vegetação, no início do período de planejamento;

NA : Número de alimentadores do sistema de distribuição;

$T$ : Horizonte de planejamento para efetuar a manutenção da vegetação sob a rede de distribuição;

$T R_{k}$ : Número de transformadores de MT/BT no alimentador $k$;

$P(k, t r)$ : Potência nominal do transformador $\operatorname{tr}(\mathrm{MT} / \mathrm{BT})$, do alimentador $k$;

$D(k, t r)$ : Demanda do transformador $\operatorname{tr}$ ( MT/BT), do alimentador $k$;

$D R_{k} \%, D I_{k} \%, D C_{k} \%$ : Porcentagem das demandas máximas residenciais, industriais e comerciais, respectivamente; do alimentador $k$; 
$\lambda(k, t)$ : taxa de falha do alimentador $k$, para o período $t$;

$r(k, t)$ : Variável de decisão binária, onde 1 significa que realiza a manutenção e 0 que não realiza a manutenção da vegetação sob o sistema de distribuição, no alimentador $k$ no período de planejamento $t$;

$C_{\mathrm{CR}}, C_{\mathrm{CC}}, C_{\mathrm{CI}}:$ Custos de interrupções de cargas residenciais, comerciais e industriais, respectivamente ( $\mathrm{R} \$ / \mathrm{kVA})$;

$l(k, t)$ : Comprimento do alimentador $k$ do sistema de distribuição que dever passar por manutenção no período $t$;

$C_{\text {esp }}$ : Recursos financeiros disponíveis pela empresa para a manutenção da vegetação no horizonte de planejamento considerado;

$L_{\text {esp }}^{t}$ : Comprimento máximo que é possível fazer manutenção no período de planejamento $t$, devido às limitações de equipes de manutenção;

$C_{\text {man }}$ : Custo da manutenção;

FEC : Frequência equivalente de interrupções por consumidor;

MF : Meta estabelecida para o FEC no período de planejamento.

O modelo matemático dado pelo conjunto de equações (2)-(6) fornece resultados que indicam ao planejador em quais alimentadores e quando deve ser realizada a poda das árvores. Note que a variável de decisão deste modelo é a variável binária $r(k, t)$, que está relacionada com a decisão de efetuar a manutenção (1), ou não (0) da vegetação sob o alimentador $k$ em uma determinada estação do ano t. A restrição (3) garante que o programa de manutenção no período de planejamento obtido através do modelo não ultrapasse o montante de recursos financeiros destinados pela empresa distribuidora no período. A restrição (4) garante que a disponibilidade das equipes de manutenção da empresa seja respeitada. A restrição (5) garante a confiabilidade do sistema de distribuição de energia, respeitando as metas estipuladas pelas agências reguladoras, no caso nacional a Agência Nacional de Energia Elétrica (ANEEL). A equação (6) fornece o custo da energia não distribuída no período anterior ao primeiro período do horizonte de planejamento. A seguir detalham-se algumas dessas restrições.

\subsubsection{Recursos Financeiros Disponíveis}

Os recursos financeiros disponíveis pela empresa de distribuição para a manutenção da vegetação devem ser compatíveis com o tempo planejado para ser realizada esta manutenção. Se os recursos não forem suficientes para executar a manutenção no tempo planejado, devem-se analisar os custos sociais e financeiros da não realização da manutenção para a concessionária e seus clientes. Esta análise permite avaliar qual é a melhor forma para a empresa investir seus recursos financeiros de modo a não ocorrer interrupções no sistema elétrico devido ao crescimento da vegetação.

É necessário conhecer, ou pelo menos estimar, a relação entre o capital investido e a expectativa de retorno destes investimentos sobre os resultados nos índices de confiabilidade do sistema elétrico.

\subsubsection{Disponibilidade das Equipes}

Para que o programa de manutenção da vegetação funcione é necessária a colaboração de equipes competentes e preparadas para cuidar do planejamento, do plantio e da manutenção da arborização, com responsabilidades perfeitamente definidas, formação adequada, experiência e treinamentos periódicos. Pessoas em geral ou arboristas sem qualificação para trabalhar junto a essas redes não estão habilitadas a realizar a poda de galhos de árvores próximas aos sistemas elétricos.

As redes elétricas primárias apresentam níveis de tensão, geralmente, muito maiores que as utilizadas em equipamentos domésticos. Tanto as redes primárias como as secundárias apresentam potencial para matar ou machucar severamente pessoas que façam contato com elas, seja direta ou indiretamente. Assim, é essencial que apenas aqueles profissionais devidamente treinados nos procedimentos e riscos associados com a arboricultura junto às redes elétricas executem serviços de manejo da vegetação.

Desta forma, no modelo matemático, a disponibilidade das equipes de manutenção (Homens vs. Horas) devidamente treinados para executar tarefas de manutenção da vegetação sob a rede é um dado limitante para se realizar a poda da vegetação urbana.

\subsubsection{Medidas de Confiabilidade de Sistemas de Distribuição}

A confiabilidade do sistema de distribuição pode ser medida através de indicadores que representam o grau de disponibilidade do sistema para o consumidor (Billinton and Billinton, 1989). Os principais indicadores usados internacionalmente são o System Average Interruption Frequency Index (SAIFI) e o System Average Interruption Duration Index (SAIDI) (IEEE Std. 1366, 2003).

Segundo as normas brasileiras, para sistema de distribuição 
de energia elétrica, os indicadores mais comuns são: a frequência equivalente de interrupções por consumidor (FEC), que corresponde ao SAIFI, e a duração equivalente de interrupções por consumidor $(D E C)$, que corresponde ao SAIDI. O índice FEC representa uma média ponderada de interrupções no fornecimento de energia elétrica durante o período, o índice $D E C$ indica a duração média, em horas, das interrupções no fornecimento de energia.

Na formulação do modelo matemático, adota-se o FEC para avaliar a confiabilidade do sistema, uma vez que o mesmo pode ser definido por unidades consumidoras e considera interrupções com duração maior ou igual a 3 (três) minutos. O FEC é calculado através das taxas de faltas (Reis et al., 2008):

$$
F E C=\frac{\sum_{k=1}^{N A} \lambda_{k} \cdot N C_{k}}{N C T}
$$

sendo:

$N A$ : número de alimentadores do sistema;

$\lambda_{k}$ : taxa de falta do alimentador $k$;

$N C_{k}$ : número de consumidores do alimentador $k$;

$N C T$ : número total de consumidores do sistema.

\section{TÉCNICA DE SOLUÇÃO}

Para a solução do modelo de otimização (2)-(6) podem ser pesquisados tanto os algoritmos de otimização clássica existentes para a solução de problemas de programação binária, assim como técnicas heurísticas e metaheurísticas. Para mostrar a validade das soluções fornecidas por este modelo através de testes com um sistema de distribuição real, propõe-se para sua solução um algoritmo genético de Chu-Beasle $(A G C B)$. Este algoritmo foi escolhido por ser de fácil implementação, apresentar bom desempenho computacional e ser adequado para a solução de problemas restritos. $\mathrm{O}$ uso deste algoritmo também propicia independência da metodologia de solução do modelo dispensando o uso de softwares comerciais. Este tipo de AG difere dos algoritmos genéticos tradicionais no processo de substituição dos elementos da população. No AG tradicional ocorre durante as gerações a substituição de todos (ou quase todos) os elementos da população através dos operadores genéticos de recombinação e mutação e, geralmente, não é efetuada a verificação da diversidade. No $A G C B$ substitui-se, em cada iteração, apenas um elemento da população corrente. Nessa proposta busca-se facilitar duas estratégias cruciais no desempenho do algoritmo: a) Permite produzir descendentes melhorados usando um processo de otimização local do descendente gerado;

b) Permite um controle absoluto da diversidade dos elementos da população corrente.

Para atender a estes requisitos, o AG de Chu-Beasley possui a seguinte estrutura básica:

i. Gera-se a população inicial aleatoriamente;

ii. Realiza-se a seleção por torneio com $k=2$ (dois indivíduos);

iii. Depois de realizar a seleção, realiza-se a recombinação entre os dois pais. Esta recombinação é realizada em quatro pontos, um para cada trimestre do ano (Figura 2);

iv. Escolhe-se apenas o indivíduo que possui a melhor função objetivo;

v. Implementa-se a mutação no indivíduo selecionado no item iv;

vi. Atualiza-se a população corrente: o descendente gerado pode ser incorporado à população corrente verificando-se as seguintes condições:

a. Se o descendente possuir a mesma configuração da população corrente ele é descartado. Este procedimento preserva a diversidade da população;

b. Se o descendente for infactível, e se na população corrente existem topologias infactíveis, o descendente gerado substituirá o indivíduo que possui maior valor de infactibilidade. Se na população corrente não existir indivíduos mais infactíveis que o descendente gerado o descendente é descartado;

c. Se o descendente é factível, deve-se substituir o indivíduo com maior infactibilidade por este descendente. $\mathrm{Se}$ todos os indivíduos da população são factíveis então o descendente gerado deve substituir o indivíduo com pior qualidade comparada caso ele exista na população. Caso contrário o descendente é descartado.

vii. Se for realizado um número preestabelecido de gerações, para-se o ciclo do AG. Caso contrário volta-se ao passo ii. 


\subsection{Codificação}

O esquema de representação (cromossomo) utilizado na codificação do problema é o representado pela Figura 2. Observa-se nesta figura que cada bit/gene é associado a um alimentador $k$ da rede de distribuição sob estudo. Cada indivíduo (configuração) é dividido em trimestre do ano formando, assim, um vetor que contém informações sobre onde e quando deve ser realizada a manutenção da vegetação no decorrer de um ano. Neste esquema de representação o bit "1" indica onde deve ser efetuada a manutenção da vegetação no alimentador $k$.

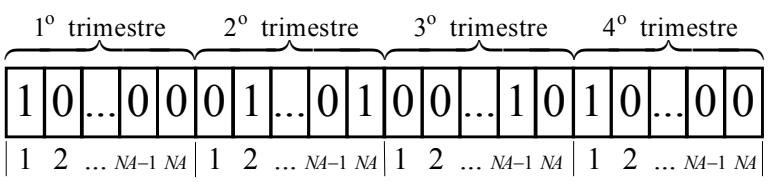

Figura 2: Esquema de Codificação.

\section{TESTES E RESULTADOS}

Devido à falta de informações sobre as espécies de vegetação que se encontram sob a rede de distribuição da Figura 3, optou-se por não trabalhar com informações concernentes às espécies de vegetação que se encontram sob cada alimentador. Dessa maneira, a equação de taxa de faltas é obtida diretamente através do modelo de curvas de dispersão não lineares e, desta forma, não sendo necessário nenhum modelo para relacionar o crescimento da vegetação às taxas de faltas.

Para cada um dos 27 alimentadores do sistema de distribuição real da Figura 3, considera-se que existe uma equação que melhor ajusta o comportamento das taxas de faltas dos anos anteriores e estima da forma mais precisa possível as taxas de faltas para o horizonte de planejamento (12 meses). Nos estudos foi considerada apenas a área urbana atendida por cada um dos alimentadores.

\subsection{Obtenção das Equações das Taxas de Faltas}

Os dados históricos das taxas de faltas dos alimentadores do sistema da Figura 3 foram manipulados no software $M A T L A B$ para gerar, para cada um deles, uma função não linear (Equação (1)) que melhor representa a relação das taxas de faltas devido à vegetação, para cada mês do horizonte de planejamento.

As equações de taxas de faltas para cada alimentador foram obtidas utilizando-se a função CFTOOL (Curve Fitting Tool), que é uma ferramenta de regressão de funções do MATLAB.
Esta ferramenta computacional possibilita analisar, dentre as diversas funções, aquelas que melhor se ajustam com o período em meses em função das taxas de faltas obtidas do sistema de distribuição de energia elétrica.

Para fazer os ajustes das taxas de faltas utilizou-se uma curva de distribuição Gaussiana, muito comum no tratamento estatístico de informações em todas as áreas do conhecimento. A equação que define a curva Gaussiana utilizada para representar as equações das taxas de faltas para cada alimentador deste sistema é dada pela equação geral:

$$
f(x)=\sum_{i=1}^{n} a_{i} \cdot e^{\left[-\left(\frac{x-b_{i}}{c_{i}}\right)^{2}\right]}
$$

sendo:

$n$ : Números de picos da gaussiana;

$a_{i}:$ Altura do pico $i$

$b_{i}$ : Posição de máximo (centro da gaussiana) do pico $i$;

$c_{i}:$ Largura do pico $i$.

Desta forma, para cada um dos 27 alimentadores do sistema de distribuição real da Figura 3, considera-se que existe uma equação que melhor ajusta o comportamento das taxas de falta dos anos anteriores e estima da forma mais precisa possível as taxas de faltas para os próximos 12 meses. Para exemplificar, a seguir estão as equações que representam as taxas pelo tempo para alguns alimentadores deste sistema teste. A base de dados utilizada e as equações das taxas de faltas para todos os alimentadores podem ser encontradas em (Apolinário, 2009).

Alimentador P1-A8:

$$
\begin{aligned}
\lambda_{6}(x) & =4,836 \cdot 10^{-5} \cdot e^{\left[-\left(\frac{x-11}{0,6519}\right)^{2}\right]}+4,557 \cdot 10^{-5} \cdot e^{\left[-\left(\frac{x-1}{0,9656}\right)^{2}\right]} \\
& +3,642 \cdot 10^{-5} \cdot e^{\left[-\left(\frac{x-9}{0,7209}\right)^{2}\right]}+2,075 \cdot 10^{-5} \cdot e^{\left[-\left(\frac{x-4}{1,539}\right)^{2}\right]} \\
& +3,1372 \cdot 10^{-5}
\end{aligned}
$$

Alimentador P3-A7:

$$
\begin{aligned}
& \lambda_{12}(x)=0,0001886 \cdot e^{\left[-\left(\frac{x-2}{1,092}\right)^{2}\right]}+7,996 \cdot 10^{-5} \cdot e^{\left[-\left(\frac{x-4}{1,716}\right)^{2}\right]} \\
& \quad+8,6323 \cdot 10^{-5}
\end{aligned}
$$

$$
\begin{aligned}
& \text { Alimentador P4-A10: } \\
& \begin{aligned}
\lambda_{21}(x) & =0,0001702 \cdot e^{\left[-\left(\frac{x-10}{0,7045}\right)^{2}\right]}+8,314 \cdot 10^{-5} \cdot e^{\left[-\left(\frac{x-1}{0,8479}\right)^{2}\right]} \\
& +8,628 \cdot 10^{-7} \cdot e^{\left[-\left(\frac{x-5}{31,1}\right)^{2}\right]}+4,4577 \cdot 10^{-5}
\end{aligned}
\end{aligned}
$$

Alimentador P5-A6:

$$
\begin{gathered}
\lambda_{27}(x)=3,086 \cdot 10^{-5} \cdot e^{\left[-\left(\frac{x-3}{1,984}\right)^{2}\right]}+3,287 \cdot 10^{-5} \cdot e^{\left[-\left(\frac{x-12}{1,613}\right)^{2}\right]} \\
\quad+2,4045 \cdot 10^{-5}
\end{gathered}
$$




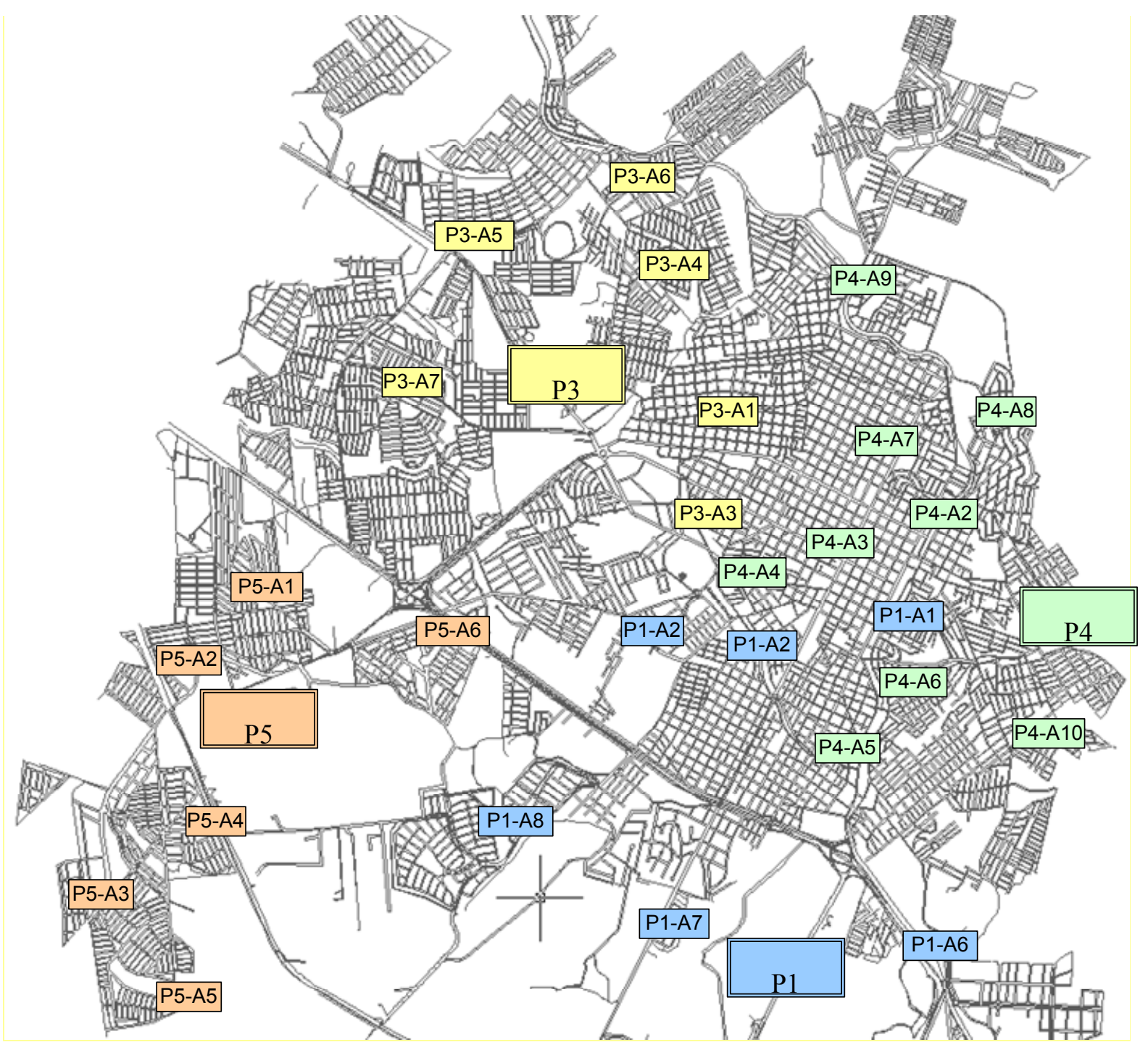

Figura 3: Sistema teste real.

\subsection{Condições de Testes}

Considerando-se que interrupções na rede de distribuição devido à vegetação sejam de $22 \%$ do valor 7 da meta $F E C$ estabelecidas pela ANEEL para o ano de 2009 (ANEEL, 2010), nas simulações realizadas utiliza-se um valor de FEC devido à vegetação igual a 2. É importante notar que se trata da proposta de uma técnica que está apoiada em algumas considerações práticas e dados históricos para programar a manutenção e não se consideram diretamente fatores meteorológicos, intempéries e outros aspectos físicos que fogem ao controle do homem. Desta forma, é importante ressaltar que o setor de planejamento das empresas, durante o procedimento de tomada de decisões, deve fazer uma análise criteriosa sob os aspectos econômicos, físicos e operacionais das propostas fornecidas pela ferramenta computacional desenvolvida a partir da metodologia proposta. Outros dados utilizados nos testes são:

- O custo de manutenção está em torno de $\mathrm{R} \$ 17,40$ por árvore. Por este custo entende-se que a empresa prestadora deste tipo de serviço deve podar as árvores e retirar os galhos das vias públicas. Como não é possível ter o número exato de árvores sob cada alimentador, optou-se por trabalhar com o custo por metro linear de rede, considerando-se uma média de 8 árvores para cada 100 metros, obtendo desta forma o custo médio de $\mathrm{R} \$ 1,39$ por metro de rede.

- A disponibilidade da equipe é dividida em 4 trimestres, sendo fixada que a disponibilidade da equipe de fazer 
a manutenção é de 171.900 metros lineares de rede por trimestre.

- O valor anual disponibilizado pela empresa para ser executada a manutenção da vegetação é de $\mathrm{R} \$ 950.000,00$.

- Os custos reais da energia não suprida (ENS) não são encontrados de forma organizada e confiável na literatura. Para contornar este problema de falta de dados reais e realizar testes com a metodologia proposta usando dados mais coerentes com a realidade, considera-se que os custos da energia não suprida representam, aproximadamente de 10 a $20 \%$ o valor do custo da manutenção da vegetação. Pode-se afirmar que supor o intervalo de variação do custo da ENS entre 10 e $20 \%$ do valor do custo da manutenção da vegetação está de acordo com dados estatísticos obtidos através de pesquisas em arquivos de dados encontrados no setor de planejamento e manutenção das concessionárias de distribuição.

Os parâmetros utilizados para simulação com o $A G C B$ são dados na Tabela 1. Estes parâmetros foram obtidos através de testes exaustivos com o programa desenvolvido usando os dados do sistema real sob estudo. Como o objetivo deste trabalho é a proposta de um modelo de planejamento de podas da vegetação sob a rede de distribuição, os parâmetros de controle do $A G C B$ foram ajustados para este sistema teste através de tentativas e erros. Para ajustar os parâmetros do $A G C B$ foram executadas 50 simulações, usando uma população inicial gerada aleatoriamente. Para cada um dos testes permitiu-se um número grande de iterações, de forma tal que o $A G C B$ fornecesse sempre os mesmos valores otimizados para a função objetivo. No caso do problema sob análise este comportamento do $A G C B$ de encontrar sempre o valor otimizado do problema deve-se à característica matemática do modelo que apresenta restrições lineares e variáveis de decisão binárias.

Tabela 1: $A G C B$ - Parâmetros de controle.

\begin{tabular}{|c|c|c|c|}
\hline População & $\begin{array}{l}\text { Número } \\
\text { máximo } \\
\text { gerações }\end{array}$ & $\begin{array}{c}\text { Taxa de } \\
\text { recombi- } \\
\text { nação }\end{array}$ & $\begin{array}{c}\text { Taxa de } \\
\text { Mutação }\end{array}$ \\
\hline 800 & 2000 & 1,0 & 0,1 \\
\hline
\end{tabular}

\subsection{Resultados dos Testes}

Para verificar a qualidade das soluções obtidas através da implementação computacional da metodologia proposta, foram realizados 2 testes.
Teste 1: A Meta estabelecida para o FEC é igual a 0, a qual representa uma condição fictícia, uma vez que do ponto de vista prático, não existe $F E C$ igual a 0 para interferência da vegetação, caso a mesma exista sob a rede. Na Tabela 2 estão apresentados os valores encontrados para esta condição de teste, sendo:

$\mathrm{CENS}_{0}$ : Custo da energia não suprida do período anterior ao início do programa de podas;

$\mathrm{CENS}_{1}$ : Custo da energia não suprida do $1^{\circ}$ trimestre de manutenção;

$\mathrm{CENS}_{2}$ : Custo da energia não suprida do $2^{\circ}$ trimestre de manutenção;

$\mathrm{CENS}_{3}$ : Custo da energia não suprida do $3^{\circ}$ trimestre de manutenção.

$\mathrm{CENS}_{4}$ : Custo da energia não suprida do $4^{\circ}$ trimestre de manutenção.

Comp. de Man. (m) : Comprimento de manutenção do período;

Custo de Man. (R\$) : Custo de manutenção do período.

Na Figura 4a apresenta-se o cromossomo da solução proposta e na Figura 4 b apresenta-se o tempo desde a última atividade de poda para cada trimestre do ano para este teste.

Teste 2: A meta estabelecida para o FEC devido à interferência da vegetação é igual a 2. A restrição de confiabilidade do sistema, equação (5), permite que ocorra interrupção do serviço de fornecimento sem que a concessionária de energia seja multada, pois os desligamentos acontecem de modo que a restrição da confiabilidade do sistema não seja violada. Para avaliar de forma indireta esta restrição, consideram-se que alimentadores com manutenção da vegetação realizada num período inferior a 9 meses não causam impacto nos indicadores do índice FEC. Na Tabela 3 apresentam-se valores encontrados para esta condição de teste. Na Figura 5 a apresenta-se o cromossomo da solução proposta e na Figura 5 b apresenta-se o tempo desde a última atividade de poda para cada trimestre do ano para este teste.

Comentários dos testes: Analisando-se os resultados das Tabelas 2 e 3 verifica-se a coerência dos resultados fornecidos pela metodologia proposta. Para um problema mais restritivo com relação à confiabilidade obtém-se custo total maior do que para a condição menos restritiva de meta de confiabilidade. $\mathrm{O}$ aumento do valor da função objetivo é devido à componente dos custos de manutenção visando minimizar os custos da ENS, que praticamente deveriam ser nulos, mas apresenta este valor devido ao período anterior ao 
Tabela 2: Valores encontrados pelo $A G C B . F E C=0$.

\begin{tabular}{|c|c|c|c|c|c|c|c|}
\hline \multicolumn{8}{|c|}{ Períodos de Manutenção } \\
\hline & & \multicolumn{3}{|c|}{$1^{\circ}$ Trimestre } & \multicolumn{3}{|c|}{$2^{\circ}$ Trimestre } \\
\hline $\begin{array}{c}\text { Custo } \\
\text { Total }(\mathrm{R} \$)\end{array}$ & $\begin{array}{c}\mathrm{CENS}_{0} \\
(\mathrm{R} \$)\end{array}$ & $\begin{array}{c}\mathrm{CENS}_{1} \\
(\mathrm{R} \$)\end{array}$ & $\begin{array}{l}\text { Comp. de } \\
\text { Man. (m) }\end{array}$ & $\begin{array}{c}\text { Custo de } \\
\text { Man. }(\mathrm{R} \$)\end{array}$ & $\begin{array}{c}\mathrm{CENS}_{2} \\
(\mathrm{R} \$)\end{array}$ & $\begin{array}{l}\text { Comp. de } \\
\text { Man. (m) }\end{array}$ & $\begin{array}{c}\text { Custo de } \\
\text { Man. (R\$) }\end{array}$ \\
\hline $879.400,54$ & $98.124,39$ & - & $172.493,15$ & $239.765,48$ & - & $141.996,56$ & $197.375,22$ \\
\hline & & \multicolumn{3}{|c|}{$3^{\circ}$ Trimestre } & \multicolumn{3}{|c|}{$4^{\circ}$ Trimestre } \\
\hline $\begin{array}{c}\text { Custo } \\
\text { Total }(\mathrm{R} \$) \\
\end{array}$ & $\begin{array}{c}\mathrm{CENS}_{0} \\
(\mathrm{R} \$)\end{array}$ & $\begin{array}{c}\mathrm{CENS}_{3} \\
(\mathrm{R} \$)\end{array}$ & $\begin{array}{l}\text { Comp. de } \\
\text { Man. (m) }\end{array}$ & $\begin{array}{c}\text { Custo de } \\
\text { Man. }(\mathrm{R} \$)\end{array}$ & $\begin{array}{c}\mathrm{CENS}_{4} \\
(\mathrm{R} \$)\end{array}$ & $\begin{array}{l}\text { Comp. de } \\
\text { Man. (m) }\end{array}$ & $\begin{array}{c}\text { Custo de } \\
\text { Man. (R\$) }\end{array}$ \\
\hline $879.400,54$ & $98.124,39$ & - & $146.901,43$ & $204.192,99$ & - & $100.678,03$ & $139.942,46$ \\
\hline
\end{tabular}

\begin{tabular}{|c|c|c|c|c|c|c|c|c|c|c|c|c|c|c|c|c|c|c|c|c|c|c|c|c|c|c|c|}
\hline 1 & 0 & 0 & 1 & 1 & 1 & 0 & 0 & 0 & 0 & 0 & 0 & 1 & 0 & 0 & 1 & 1 & 1 & 0 & 0 & 1 & 1 & 0 & 1 & 1 & 0 & 0 & $1^{\circ}$ Trimestre \\
\hline 0 & 1 & 0 & 0 & 0 & 0 & 1 & 1 & 1 & 0 & 0 & 0 & 0 & 1 & 0 & 0 & 0 & 0 & 1 & 0 & 1 & 0 & 1 & 1 & 1 & 1 & 1 & $2^{\circ}$ Trimestre \\
\hline 1 & 0 & 1 & 1 & 0 & 0 & 1 & 1 & 1 & 1 & 1 & 1 & 0 & 0 & 1 & 0 & 0 & 0 & 0 & 0 & 0 & 0 & 0 & 0 & 0 & 0 & 0 & $3^{\circ}$ Trimestre \\
\hline 0 & 0 & 0 & 0 & 0 & 0 & 0 & 0 & 0 & 0 & 0 & 0 & 0 & 0 & 0 & 0 & 0 & 0 & 0 & 1 & 0 & 0 & 0 & 0 & 0 & 0 & 0 & $4^{\circ}$ Trimestre \\
\hline
\end{tabular}

(a) Proposta de Solução.

\begin{tabular}{|c|c|c|c|c|c|c|c|c|c|c|c|c|c|c|c|c|c|c|c|c|c|c|c|c|c|c|c|}
\hline 3 & 3 & 3 & 14 & 11 & 15 & 6 & 6 & 4 & 4 & 4 & 2 & 1 & 1 & 1 & 12 & 11 & 12 & 1 & 1 & 11 & 8 & 7 & 7 & 8 & 7 & 8 & $1^{\circ}$ Trimestre \\
\hline 3 & 6 & 6 & 3 & 3 & 3 & 9 & 9 & 7 & 7 & 7 & 5 & 3 & 4 & 4 & 3 & 3 & 3 & 4 & 4 & 3 & 3 & 10 & 3 & 3 & 10 & 10 & $2^{\circ}$ Trimestre \\
\hline 6 & 3 & 9 & 6 & 6 & 6 & 3 & 3 & 3 & 10 & 10 & 8 & 6 & 3 & 7 & 6 & 6 & 6 & 3 & 7 & 3 & 6 & 3 & 3 & 3 & 3 & 3 & $3^{\circ}$ Trimestre \\
\hline 3 & 6 & 3 & 3 & 9 & 9 & 3 & 3 & 3 & 3 & 3 & 3 & 9 & 6 & 3 & 9 & 9 & 9 & 6 & 10 & 6 & 9 & 6 & 6 & 6 & 6 & 6 & $4^{\circ}$ Trimestre \\
\hline
\end{tabular}

(b) Tempo desde a última manutenção.

Figura 4: Aumento da ENS e FEC igual a zero.

Tabela 3: Valores encontrados pelo $A G C B . F E C=2$ (Aumento do custo da $E N S$ ).

\begin{tabular}{|c|c|c|c|c|c|c|c|}
\hline \multicolumn{8}{|c|}{ Períodos de Manutenção } \\
\hline & & \multicolumn{3}{|c|}{$1^{\mathrm{o}}$ Trimestre } & \multicolumn{3}{|c|}{$2^{\circ}$ Trimestre } \\
\hline $\begin{array}{c}\text { Custo } \\
\text { Total (R\$) }\end{array}$ & $\begin{array}{c}\mathrm{CENS}_{0} \\
(\mathrm{R} \$)\end{array}$ & $\begin{array}{c}\mathrm{CENS}_{1} \\
(\mathrm{R} \$)\end{array}$ & $\begin{array}{l}\text { Comp. de } \\
\text { Man. (m) }\end{array}$ & $\begin{array}{c}\text { Custo de } \\
\text { Man. (R\$) }\end{array}$ & $\begin{array}{c}\mathrm{CENS}_{2} \\
(\mathrm{RS})\end{array}$ & $\begin{array}{l}\text { Comp. de } \\
\text { Man. (m) }\end{array}$ & $\begin{array}{l}\text { Custo de } \\
\text { Man. (R\$) }\end{array}$ \\
\hline $833.548,19$ & $98.124,39$ & - & $162.280,40$ & $225.569,76$ & - & $163.928,75$ & $227.860,96$ \\
\hline & & \multicolumn{3}{|c|}{$3^{\circ}$ Trimestre } & \multicolumn{3}{|c|}{$4^{\circ}$ Trimestre } \\
\hline $\begin{array}{c}\text { Custo } \\
\text { Total (R\$) }\end{array}$ & $\begin{array}{c}\mathrm{CENS}_{0} \\
(\mathrm{R} \$)\end{array}$ & $\begin{array}{c}\mathrm{CENS}_{3} \\
(\mathrm{R} \$) \\
\end{array}$ & $\begin{array}{l}\text { Comp. de } \\
\text { Man. (m) }\end{array}$ & $\begin{array}{c}\text { Custo de } \\
\text { Man. (R\$) }\end{array}$ & $\begin{array}{c}\mathrm{CENS}_{4} \\
(\mathrm{R} \$)\end{array}$ & $\begin{array}{l}\text { Comp. de } \\
\text { Man. (m) }\end{array}$ & $\begin{array}{c}\text { Custo de } \\
\text { Man. (R\$) }\end{array}$ \\
\hline $833.548,19$ & $98.124,39$ & - & $171.641,81$ & $238.582,12$ & $3.097,31$ & $31.230,91$ & $43.410,96$ \\
\hline
\end{tabular}




\begin{tabular}{|c|c|c|c|c|c|c|c|c|c|c|c|c|c|c|c|c|c|c|c|c|c|c|c|c|c|c|c|}
\hline 0 & 0 & 0 & 1 & 1 & 1 & 0 & 0 & 0 & 0 & 0 & 0 & 1 & 1 & 1 & 1 & 1 & 1 & 0 & 0 & 1 & 1 & 0 & 0 & 0 & 0 & 0 & $1^{\circ}$ Trimestre \\
\hline 1 & 1 & 0 & 0 & 0 & 0 & 1 & 0 & 0 & 0 & 1 & 1 & 0 & 0 & 0 & 0 & 0 & 0 & 0 & 0 & 0 & 0 & 0 & 0 & 1 & 1 & 1 & $2^{\circ}$ Trimestre \\
\hline 0 & 0 & 1 & 0 & 0 & 0 & 0 & 1 & 1 & 1 & 0 & 0 & 1 & 0 & 1 & 0 & 0 & 0 & 0 & 1 & 0 & 0 & 1 & 1 & 0 & 0 & 0 & $3^{\circ}$ Trimestre \\
\hline 0 & 1 & 0 & 1 & 0 & 0 & 0 & 0 & 0 & 0 & 0 & 0 & 0 & 1 & 1 & 0 & 1 & 0 & 1 & 0 & 0 & 0 & 1 & 1 & 0 & 1 & 0 & $4^{\circ}$ Trimestre \\
\hline
\end{tabular}

(a) Proposta de Solução.

\begin{tabular}{|c|c|c|c|c|c|c|c|c|c|c|c|c|c|c|c|c|c|c|c|c|c|c|c|c|c|c|c|}
\hline 3 & 3 & 3 & 14 & 11 & 15 & 6 & 6 & 4 & 4 & 4 & 2 & 1 & 1 & 1 & 12 & 11 & 12 & 1 & 1 & 11 & 8 & 7 & 7 & 8 & 7 & 8 & ${ }^{\circ}$ Trimest \\
\hline 6 & 6 & 6 & 3 & 3 & 3 & 0 & 9 & 7 & 7 & 7 & $\varepsilon$ & 3 & 4 & 3 & 3 & 3 & 3 & 4 & 4 & 3 & 3 & 10 & 10 & 11 & 10 & 10 & \\
\hline 3 & 3 & 9 & 6 & 6 & 6 & 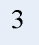 & 10 & 10 & 10 & $J$ & J & & 7 & 0 & 6 & 6 & 0 & 7 & 7 & & & 13 & 13 & & & 3 & \\
\hline 6 & 6 & 3 & 9 & 9 & 9 & & 3 & 5 & 3 & 6 & 0 & 3 & 1 & 3 & 9 & 9 & 9 & 1 & $J$ & 9 & & 3 & 3 & 0 & 6 & 3 & Tr \\
\hline
\end{tabular}

(b) Tempo desde a última manutenção.

Figura 5: Aumento da ENS e FEC igual a dois

período de planejamento. A restrição de meta de $F E C$ nula implica em um aumento de 5,5\% com relação ao custo total para a meta de $F E C$ igual a 2.

Os resultados das Tabelas 2 e 3 mostram que a alteração de metas dos indicadores de confiabilidade faz com que haja a necessidade de realocar equipes de manutenção em diferentes épocas do ano. Estes resultados indicam que é possível, dependendo das necessidades da empresa, readequar e planejar a composição das equipes de manutenção.

Na Figura 4a verifica-se que, no período de tempo sob análise, é realizada a manutenção da vegetação sob todos os alimentadores, o que permite que não haja interrupção no sistema devido à vegetação, respeitando a restrição de confiabilidade. Na Figura $4 \mathrm{~b}$ apresenta-se o tempo que a vegetação sob os alimentadores está sem manutenção, no período dos 4 trimestres sob análise, tomando-se como referência o início de cada trimestre. Neste tempo, sem que ocorra a manutenção, consideram-se as taxas de faltas históricas de cada alimentador e busca-se contemplar que o período sem manutenção não seja superior a 9 meses. Nos alimentadores cujo período sem manutenção é superior a 9 meses ocorre porque as suas respectivas taxas de faltas devido à vegetação são insignificantes. No início do primeiro trimestre os custos dos desligamentos devido à vegetação são considerados nos custos de energia não suprida inicial $\left(C_{0}\right)$.

Na Figura 5a observa-se que existem alimentadores em que não foi realizada a manutenção da vegetação no período de um ano, ou seja, os tempos de manutenção são superiores a 10 meses. Isso acontece devido ao fato de que o custo da energia não suprida $(E N S)$ estimado é bem menor do que o custo para realizar a manutenção da vegetação no alimentador, então é preferível pagar pela energia não distribuída, do que realizar a manutenção. O custo da ENS neste caso é priorizado no modelo, diferente do teste em que se prioriza a meta do FEC igual a zero. Economiza-se com a manutenção do sistema, mantendo-se a meta de $F E C$ igual a 2. A restrição de confiabilidade do sistema permite que ocorra interrupção sem que a concessionária seja multada, pois os desligamentos acontecem de modo que a restrição da confiabilidade do sistema não seja violada.

\section{CONSIDERAÇÕES FINAIS}

A partir dos resultados encontrados verifica-se a robustez e eficiência da metodologia proposta através de um plano de manutenção com a minimização dos desligamentos do serviço de fornecimento devido ao crescimento da vegetação. Para o sistema real utilizado nos testes foram desenvolvidas equações de taxas de faltas por alimentador que melhor representam as taxas de faltas históricas.

O indicador $F E C$ é uma importante ferramenta utilizada pela ANEEL para supervisionar e avaliar a continuidade da distribuição de energia elétrica das concessionárias. Neste trabalho a restrição de confiabilidade do sistema (Equação (5)) garante que a concessionária de energia não pague multas devido às interrupções provocadas pela interferência da vegetação.

É importante notar que, até o presente momento, as empresas utilizam uma metodologia empírica para estabelecer o programa de manutenção da vegetação. $\mathrm{O}$ modelo matemático proposto engloba todas as variáveis envolvidas no problema, objetivando minimizar o custo de manutenção de forma a obedecer as normas vigentes relacionadas à confiabilidade do sistema.

A técnica de solução proposta demonstra a validade do modelo e, assim como outros aspectos do problema apresentado, são temas promissores de pesquisa. O modelo 
proposto pode ser melhorado com o conhecimento dos tipos de vegetação existentes sob a rede. Este inventário da vegetação sob a rede permitiria obter modelos mais confiáveis das taxas de faltas, confrontando os modelos obtidos através de interpolações com a quantidade e o tipo de árvores existentes sob o alimentador e fazendo uma correlação entre estes dados. Com o inventário da vegetação também é possível estabelecer políticas de custos e de contratação de mão de obra, além de incluir estes aspectos no modelo.

\section{AGRADECIMENTOS}

Os autores agradecem a Empresa CAIUÁ Serviços de Eletricidade Ltda (Presidente Prudente) pela consultoria técnica e fornecimento de dados de seus sistemas de distribuição, e à CAPES, CNPq e FEPISA pelo apoio financeiro. Agradecemos a Dra. Mara Lúcia Martins Lopes do DM/FEIS/UNESP pela colaboração.

\section{REFERÊNCIAS}

ANEEL (2010). Resolução autorizativa $n^{0}$ 1589 de 07/10/2008, Disponível em http://www.aneel.gov.br/cedoc/rea20081589.pdf (último acesso 22/04/2010). Publicado em 20/10/2008 - ANEEL ( vigente).

Apolinário, L. (2009). Modelo de programação matemática para controle do crescimento da vegetação sob redes de distribuição de energia elétrica., Disponível em http://www.dee.feis.unesp.br/lapsee/papers/Tesis/Dissertacao_LilianiApolinario.pdf. Dissertação de Mestrado, Programa de Pós Graduação em Engenharia Elétrica UNESP de Ilha Solteira - SP.

Appelt, P. J. and Beard, A. (2006). Components od an effective vegetation management program., IEEE Rural Electric Power Conference. pp. B3-1 : B3-8.

Billinton, R. and Billinton, J. (1989). Distribution system reliability indices., IEEE Transactions on Power Delivery, New York. 4(1): 561-568.

CESP (1995). Guia de arborização. Companhia energética de São Paulo. 33p.

Chu, P. and Beasley, J. (1997). An genetic algorithm for the generalized assignment problem., Computers Operations Research 24(1): 17-23.

COELBA (1995). Guia de arborização urbana. Companhia de eletricidade de estado de Bahia. Euclides da Cunha: COELBA. 32 p.
CPFL (2003). Planejamento e controle da arborização na coexistência com o sistema elétrico. Companhia paulista de força e luz. São Paulo: CPFL. 19p.

ELETROPAULO (2003). Guia de arborização urbana: manual de poda. São Paulo: ELETROPAULO. 74 p.

Guikema, S., Davidson, R. and Liu, H. (2006). Statistical models of the effects of tree trimming on power system outages., IEEE Transactions on Power Delivery 21(3): 1549-1557.

IEEE Std. 1366 (2003). IEEE guide for electric power distribution reliability Índices 1366 . IEEE Power Engineering Society. Sponsored by the Transmission and Distribution Committee.

Kuntz, D., Christie, R. and Venkata, S. (2002). Optimal vegetation maintenance scheduling of overhead electric power distribution systems., IEEE Transactions on Power Delivery, New York. 17(4): 1164-1169.

Oliveira Junior, A. (1996). Redução dos custos de empresas de distribuição de energia através da adequação da arborização urbana. São Paulo: CESP, 9 p.

Pivetta, K., Demattê, M., Lima, S. and Donegá, I. (1992). Caracterização e análise da arborização de ilha solteira, sp., Congresso brasileiro sobre arborização urbana, 1; encontro nacional sobre arborização urbana. Anais, Vitória, PMV/SMMA 4: 449.

Radmer, D., Kuntz, P., Christie, R., Venkata, S. and Fletcher, R. (2002). Predicting vegetation - related rates for overhead distribution feeders., IEEE Transactions on Power Delivery. 17(4): 1170-1175.

Reis, P., Filho, C., Cavelluci, C., Zuben, F., Usberti, F., Gonzáles, J., Coelho, G. and Ferreira, H. (2008). Problema de alocação Ótima de recursos de manutenção: Formulação e estudo de caso., Anais do XL Simpósio Brasileiro de Pesquisa Operacional. João Pessoa PB, SOBRAPO pp. 448-459.

Velasco, G. (2003). Arborização viária x sistema de distribuição de energia elétrica: avaliação dos custos, estudo das podas e levantamento de problemas fitotécnicos. Dissertação (Mestrado em Agronomia) - Escola Superior de Agricultura Luiz de QueirozESALQ, Universidade de São Paulo, Piracicaba.

Wischkaemper, J., Benner, C. and Dan Russel, B. (2008). Electrical characterization of vegetation contatcts with distribution conductors - investigagtion of progressive fault behavior., IEEE Transmission and Distribution Conference and Exposition - TD . 